\title{
Tratamiento exitoso con activador funcional removible en paciente clase II esqueletal con mordida en tijera, después del peak de creci- miento puberal: caso y revisión de la literatura
}

\section{Successful treatment with removable functional activator in a skeletal class II patient with scissor bite, after pubertal growth peak: clinical case and literature review}

\author{
J. Aylwin Ramírez*, A. Hidalgo Rivas** , E. Palma Díaz**
}

\section{RESUMEN}

Introducción: En pacientes con crecimiento esqueletal clase II, el tratamiento ideal es etiológico modificando la cantidad y dirección de crecimiento mandibular. Con estos objetivos, se usan activadores funcionales (AF) que estimulan el crecimiento mandibular, redirigiéndolo posterosuperiormente a nivel condilar por medio del avance mandibular. Después del peak de crecimiento puberal, la corrección etiológica es quirúrgica. El objetivo del presente artículo es reportar un tratamiento exitoso de un paciente después de su peak de crecimiento puberal, tratado con un AF removible.

Métodos: Paciente de 13 años, 3 meses, braquifacial y con perfil facial convexo, presentaba clase Il esqueletal debido a mandíbula retrognática. El paciente tenía dentición permanente completa, clase II de Angle, resalte incisivo y sobremordida aumentados, proinclinación incisiva bimaxilar y mordida en tijera de dientes \#2.4 y \#2.5. Resultados: Después de ocho meses de tratamiento, se observó posición mandibular estable en clase I esqueletal, verificada mediante exámenes funcionales y radiográficos. Se logró resolución de la mordida en tijera. Mejoraron resalte incisivo y sobremordida, así como la proinclinación incisiva bimaxilar.

Conclusiones: El momento ideal para utilizar AF en tratamiento de clases II esqueletales es durante o ligeramente después del peak de crecimiento puberal. Sin embargo, los resultados clínicos del presente caso, permiten recomendar el avance mandibular con AF en pacientes braquifaciales clase II, a pesar de que se haya producido el peak de crecimiento puberal. En estos casos, el uso de AF está dirigido principalmente a beneficios dentarios, pero, al mismo tiempo, puede favorecer el crecimiento mandibular.

PALABRAS CLAVE: Aparatos funcionales de ortodoncia; peak de crecimiento puberal; maloclusión; mordida en tijera.

\section{ABSTRACT}

Introduction: In growing skeletal class II patients, the ideal treatment is etiological and is obtained by modifying the amount and direction of mandibular growth. With this objective in mind, functional activators (FA) are used as they stimulate growth, redirecting it at the condylar level through forward mandibular advancement. After pubertal growth peak, etiological correction is surgical.

* Cirujano Dentista, Especialista en Ortodoncia y Ortopedia Dentofacial Hospital de Gorbea,Servicio de Salud Araucanía Sur, Chile

** Programa de Especialización en Ortodoncia y Ortopedia Dentofacial

Departamento de Estomatología Pediátrica, Universidad de Talca, Talca, Chile. 
Objective:The present article aims to report a successful treatment in a patient after pubertal growth peak treated with a removable FA.

Methods: A 13 years 3 months male patient, skeletal class II due to retrognathic mandible, brachyfacial and with a convex facial profile. The patient had complete permanent dentition, Angle class II, increased overjet and overbite. Maxillary and mandibular incisive proclination. Scissor bite of teeth \#2.4 and \#2.5.

Results: Treatment started using a FA for one year after first evaluation. Following eight months of treatment, stable mandibular position was observed in skeletal class I, verified by functional and radiographic examinations. Resolution of scissor bite was accomplished. Overjet and overbite, and bimaxillary incisive proclination were improved.

Conclusions: The ideal time to use FAs for skeletal management in skeletal class II is during, or slightly after peak pubertal growth. However, the clinical results of present case, allows recommending the forward mandibular advancement in brachyfacial skeletal class II patients, even though pubertal growth peak had occurred. In these cases, the use of FA is primarily aimed at the dental benefits, but, at the same time, favoring mandibular growth.

KEY WORDS: Orthodontic appliances, functional; pubertal growth peak;malocclusion; scissor bite.

Fecha de recepción: 31 de octubre de 2019

Fecha de aceptación: 25 de noviembre de 2019

J. Aylwin Ramírez, A. Hidalgo Rivas, E. Palma Díaz. Tratamiento exitoso con activador funcional removible en paciente clase II esqueletal con mordida en tijera, después del peak de crecimiento puberal: caso y revisión de la literatura. 2020; 36, (4): xxx-xxx.

\section{INTRODUCCIÓN}

Las maloclusiones en relación con clase II esqueletal son causadas con mayor frecuencia por una mandíbula retrognática ${ }^{(1)}$ y ocurren en un tercio de la población ${ }^{(2)}$. Para los pacientes con crecimiento de clase II con mandíbula retrognática, el medio ideal de corrección es etiológico, y se debe intentar modificar la cantidad o la dirección del crecimiento mandibular ${ }^{(3,4)}$.

Una de las alternativas de tratamiento para la clase II esqueletal son los activadores funcionales (AF). Los AF generan un avance mandibular ${ }^{(2,5,6)}$ para corregir una mandíbula retrognática, obteniendo una correcta relación sagital mandibular sin proinclinar los incisivos inferiores ${ }^{(5,6)}$.

En los tratamientos clase II esqueletales, una ventaja del uso de AF en comparación con una alternativa quirúrgica es el hecho de trabajar con el propio estímulo de crecimiento del paciente ${ }^{(6)}$. Con los AF es posible modificar y redirigir el crecimiento condilar ${ }^{(5)}$. El tratamiento con AF es una opción menos invasiva y menos costosa para el paciente, en comparación con la alternativa quirúrgica. El uso de $\mathrm{AF}$ ayuda a restaurar el equilibrio muscular, a la vez que coordina las relaciones espaciales entre maxilar y mandíbula ${ }^{(5)}$.
El uso de AF removibles para tratar la clase II esqueletal en pacientes en crecimiento ha demostrado ser eficaz ${ }^{(7)}$. Sin embargo, en pacientes en etapas posteriores al peak de crecimiento puberal, los resultados de la terapia con AF siguen siendo poco claros y de pronóstico incierto ${ }^{(8)}$. Esto se debe a que el estímulo muscular producido por los AF potencia los mecanismos biológicos que determinan un crecimiento mandibular activo ${ }^{(3,4)}$.

Se reporta un caso de tratamiento exitoso usando un AF removible en un paciente clase II esqueletal un año después del peak de crecimiento puberal, con mordida en tijera, resalte incisivo, sobremordida aumentados y proinclinación incisiva bimaxilar.

\section{Diagnóstico}

Paciente de 13 años y 3 meses, asistió a la Clínica Odontológica del Programa de Especialización en Ortodoncia y Ortopedia Dentofacial de la Universidad de XX para ser tratado por anomalías dentomaxilares. El paciente reportó el uso de biberón hasta los 7 años. El examen extraoral mostró leves alteraciones posturales, simetría facial, perfil facial convexo y ángulo nasolabial normal. En la evaluación intraoral se observó dentición 


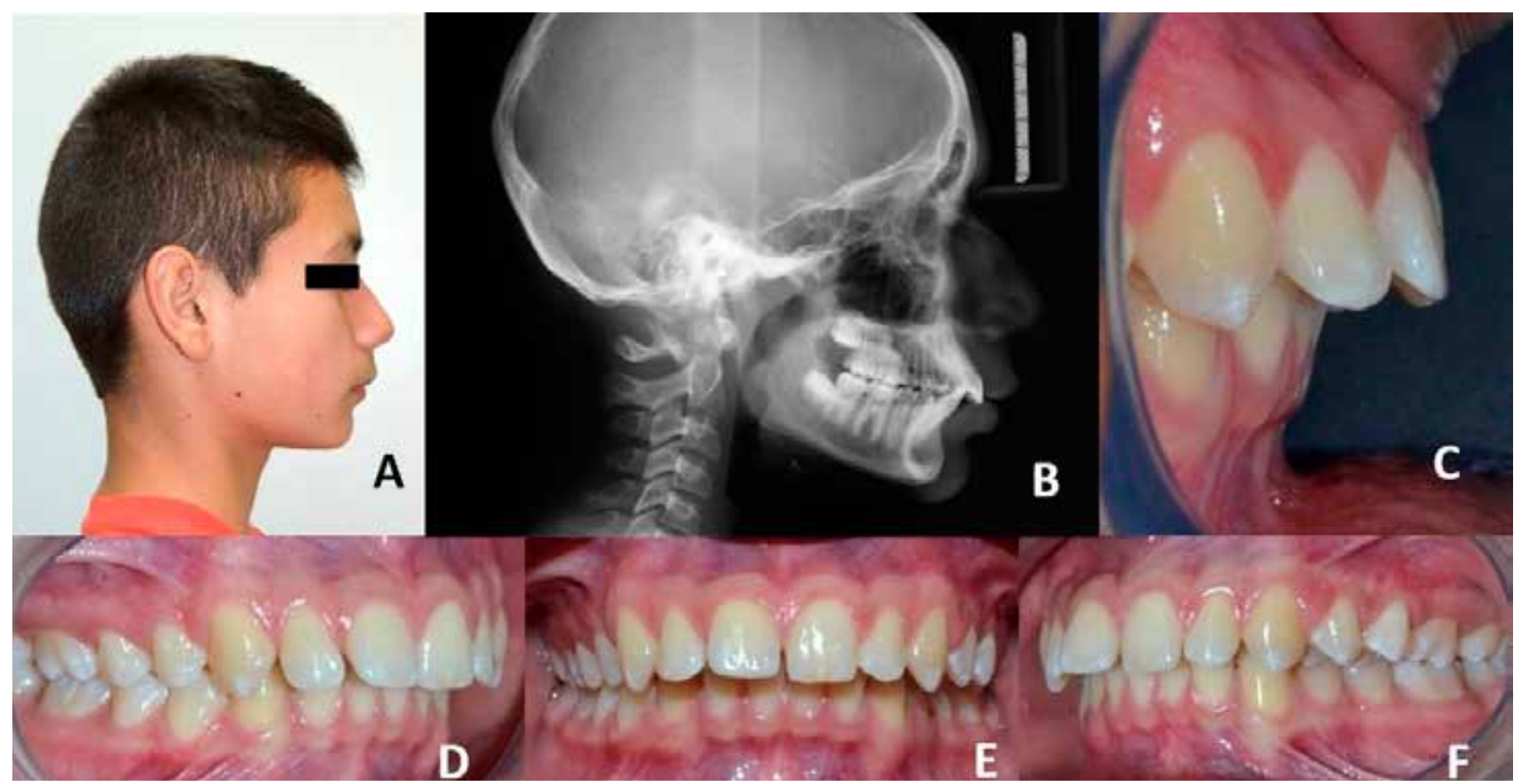

Figura 1. Fotografías iniciales. Arcos dentarios permanentes completos, con forma irregular y descoordinados. A: Perfil facial convexo (anterior, retroinclinado). B: Telerradiografía lateral de cráneo. Índice de Maduración Vertebral en etapa 3, clase II esqueletal C: Resalte de 7 milímetros y sobremordida de 5 milímetros. D: Clase II molar y canina bilaterales. E: Línea media inferior desviada 0,5 milímetros a la izquierda. F: Mordida en tijera de dientes \#2.4 y \#2.5 con resalte lateral de 1 milímetro.

permanente completa. Los arcos dentales superior e inferior tenían forma irregular y no estaban coordinados entre sí. Las relaciones molares y caninas fueron bilateralmente de clase II. A nivel de incisivos, se observó un resalte de $7 \mathrm{~mm}$ y una sobremordida de $5 \mathrm{~mm}$. Había una mordida en tijera en los dientes \#2.4 y \#2.5. Se observó una discrepancia esquelético-dental de $1 \mathrm{~mm}$ en el arco superior y no hubo discrepancia en el arco inferior. Se observó proinclinación de incisivos maxilares y mandibulares, así también periodonto fino en el área canina mandibular (Figura 1). No se encontraron alteraciones de las articulaciones temporomandibulares (ATM).

En la telerradiografía craneal lateral, se evidenció un Índice de Maduración Vertebral (VMI, del inglés:Vertebral Maturity Index) en Etapa Cervical 3 (CS3), indicando que el paciente estaba en el peak de crecimiento puberal ${ }^{(13)}$. La evaluación cefalométrica de la telerradiografía craneal lateral indicó una relación de clase II esqueletal por mandíbula retrognática, crecimiento rotacional anterior mandibular y biotipo braquifacial. Por motivos personales, el paciente pudo empezar su tratamiento al año después de este diagnóstico.

\section{Plan de tRatamiento}

Los objetivos ideales del tratamiento fueron: 1) En el aspecto esqueletal, corregir la relación esqueletal clase II. 2) En el aspecto funcional: normalización de la musculatura. 3) En el aspecto dental: obtener la clase I, relación canina y valores adecuados de la inclinación, resalte y sobremordida de incisivos. Corregir la mordida en tijera en los dientes \#2.4 y \#2.5 y corregir la forma y coordinación de arcos dentarios.

Para cumplir con los objetivos del tratamiento, se propuso inicialmente un tratamiento ortodóncico-quirúrgico. Sin embargo, no se obtuvo el consentimiento de los padres ni el asentimiento del paciente. Por lo tanto, se planificó una corrección inicial con un AF removible y, posteriormente, un tratamiento de ortodoncia con aparatos fijos.

\section{Progreso del tRatamiento}

El AF se confeccionó con una mordida constructiva, con un avance mandibular de $5 \mathrm{~mm}$ y centrando la línea media dental inferior con respecto a la 
línea media dental superior y facial. El AF tenía ansas vestibulares maxilares y mandibulares, un coffin maxilar hecho en acero inoxidable $1 \mathrm{~mm}$ y resortes vestibulares de acero inoxidable $0,8 \mathrm{~mm}$ en relación a los dientes \#2.4, \#2.5 y \#2.6 (Figura $2-\mathrm{A})$; y para superficies linguales de dientes \#3.4, \#3.5 y \#3.6 (Figura 2-B).

El uso de AF fue indicado durante el día, con la remoción sólo cuando el paciente comía. El paciente fue controlado mensualmente para evaluar el progreso del tratamiento y activar los resortes del AF para corregir la mordida en tijera. Además, se realizó desgaste en áreas del acrílico que interferían con el movimiento dental deseado, para permitir la coordinación en los sectores laterales de los arcos dentales. Se mantuvo un monitoreo periódico de las ATM mediante controles clínicos.

Después de ocho meses de tratamiento (Figura 3), se observó una posición mandibular estable, con resolución total de la mordida en tijera y mejora parcial de la alineación y relaciones verticales y sagitales de los arcos dentales. Se indicó una telerradiografía craneal lateral para evaluar el cre-

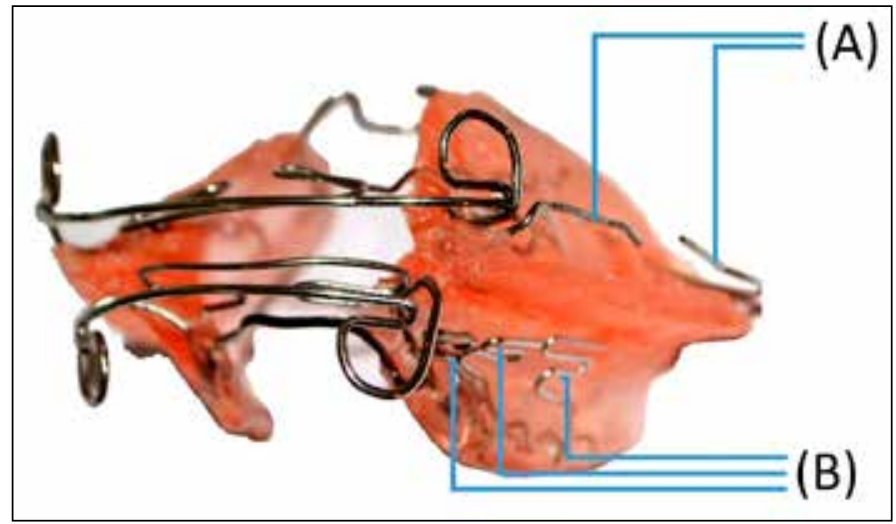

Figura 2. Activador funcional utilizado. Presenta resortes (A) para mover hacia palatino los dientes \#2.4 y \#2.5. Además, posee resortes en " $\mathrm{S}$ " (B) para mover hacia vestibular los dientes \#3.4, \#3.5 y \#3.6.

cimiento condilar y comparar los valores cefalométricos con los de inicio del tratamiento.

\section{RESULTADOS}

Al comparar los análisis cefalométricos iniciales y el estadio ortopédico a los 8 meses de tratamiento (Tabla 1), se observó que el paciente, que al prin-

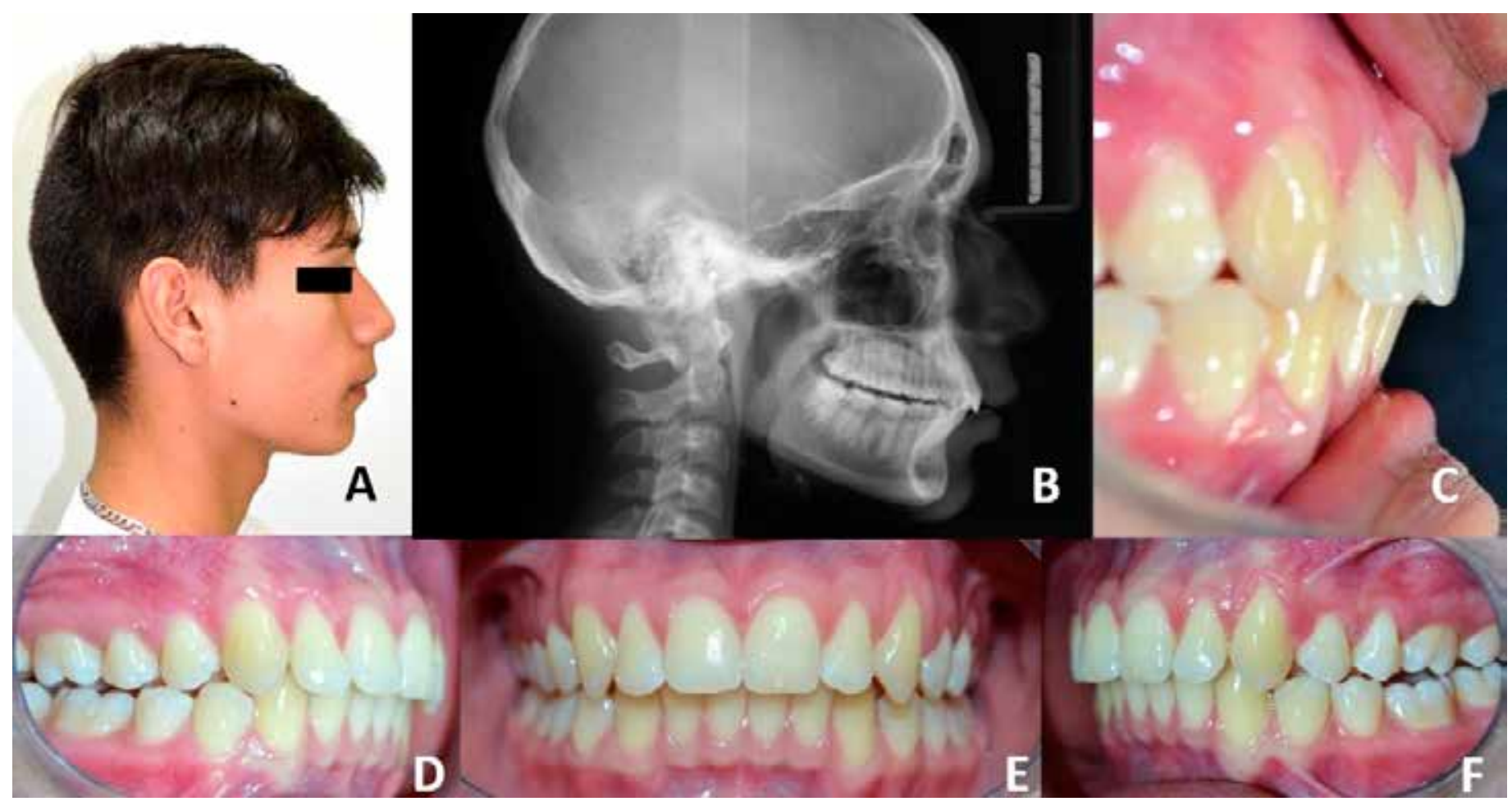

Figura 3. Fotografías posteriores a la etapa ortopédica. Arcos dentarios permanentes completos, ovalados y coordinados. A: Perfil facial convexo (anterior, recto). B: Telerradiografía lateral de cráneo. Índice de Maduración Vertebral en etapa 3, clase I esqueletal. C: Resalte de 3 milímetros y sobremordida de 2,5 milímetros. D: Clase I molar y canina bilaterales. E: Línea media inferior desviada 0,5 milímetros a la izquierda. F: Mordida en tijera de dientes \#2.4 y \#2.5 resuelta. 
cipio era clase II esqueletal, había alcanzado la clase I esqueletal. Esta armonía ósea se evidenció por diferentes valores, tales como el ángulo ANB y el Wits Appraisal. Además, se observó etapa CS4 del VMI, confirmando el final del peak de crecimiento puberal. La manipulación mandibular, palpación y auscultación de las ATM, y análisis clínicos y radiográficos -con telerradiografía craneal lateral y radiografía panorámica- mostraron salud articular.

La mordida en tijera se corrigió a nivel dentoalveolar, logrando una adecuada conformación y coordinación de los arcos dentales. También se observaron relaciones molares y caninas de clase I y línea media dental centrada.

\section{DISCUSIÓN}

El presente caso muestra un tratamiento ortopédico exitoso para un paciente clase II esqueletal, con mordida en tijera, pasado el peak de crecimiento puberal. En el momento del diagnóstico, el paciente tenía 13 años 3 meses y el VMI mostraba que la mandíbula estaba en su punto máximo de crecimiento.

Por razones personales, el paciente no pudo iniciar el tratamiento hasta un año después del diagnóstico.Cuando el paciente regresó, se evaluó la posibilidad de realizar un tratamiento ortodóncico-quirúrgico para lograr avance mandibular, una vez completado el crecimiento. Se sabe que, en el caso de la clase II esqueletal, que se encuentra al final de su peak de crecimiento puberal, la primera opción de tratamiento es ortodóncico-quirúrgica ${ }^{(8,9)}$. La opción ortodóncico-quirúrgica también se basó en la magnitud de la discrepancia esqueletal y la mordida en tijera observada en el presente caso.

La segunda opción terapéutica propuesta para el presente caso fue el tratamiento ortopédico-ortodóncico con un AF removible para clase II esqueletal. Las expectativas de éxito de esta opción dependen de la cooperación del paciente en el uso del AF. Después del crecimiento máximo, la posibilidad de mejorar el crecimiento mandibular no era buena. La mejor contribución del AF sería corregir la mordida en tijera y las malposiciones dentales, junto con las mejoras en la función muscular.
TABLA 1. Comparación de valores cefalométricos iniciales y finales de la etapa ortopédica, a los 8 meses de tratamiento.

\begin{tabular}{|c|c|c|c|c|c|}
\hline & Medidas & $\begin{array}{c}\text { Valores } \\
\text { pretratamiento }\end{array}$ & $\begin{array}{c}\text { Norma * } \\
\text { pretratamiento }\end{array}$ & $\begin{array}{c}\text { Valores } \\
\text { postratamiento }\end{array}$ & $\begin{array}{c}\text { Norma * } \\
\text { postratamiento }\end{array}$ \\
\hline \multirow{6}{*}{$\begin{array}{c}\text { Mediciones } \\
\text { Esqueletales } \\
\text { sagitales }\end{array}$} & SNA & $84^{\circ}$ & $82^{\circ}$ & $82,5^{\circ}$ & $82^{\circ}$ \\
\hline & SNB & $79^{\circ}$ & $80^{\circ}$ & $79^{\circ}$ & $80^{\circ}$ \\
\hline & $\overline{A N B}$ & $5^{\circ}$ & $2^{\circ}$ & $3,5^{\circ}$ & $2^{\circ}$ \\
\hline & Evaluación deWits & $1 \mathrm{~mm}$ & $-1 \mathrm{~mm}$ & $-1 \mathrm{~mm}$ & $-1 \mathrm{~mm}$ \\
\hline & Convexidad punto $\mathrm{A}$ & $3 \mathrm{~mm}$ & $1,1 \mathrm{~mm}$ & $2,6 \mathrm{~mm}$ & $0,8 \mathrm{~mm}$ \\
\hline & Convexidad punto $\mathrm{A}$ & $3 \mathrm{~mm}$ & $1,1 \mathrm{~mm}$ & $2,6 \mathrm{~mm}$ & $0,8 \mathrm{~mm}$ \\
\hline $\begin{array}{l}\text { Mediciones } \\
\text { Esqueletales } \\
\text { vertical }\end{array}$ & Atura facial inferior & $34^{\circ}$ & $47^{\circ}$ & $34,4^{\circ}$ & $47^{\circ}$ \\
\hline \multirow{3}{*}{$\begin{array}{c}\text { Mediciones } \\
\text { Mandibulares }\end{array}$} & $\begin{array}{l}\text { Longitud cuerpo } \\
\text { mandibular (mm) }\end{array}$ & $69 \mathrm{~mm}$ & $72,2 \mathrm{~mm}$ & $74,6 \mathrm{~mm}$ & $73 \mathrm{~mm}$ \\
\hline & $\begin{array}{l}\text { Longitud man- } \\
\text { dibular efectiva } \\
\text { Mcnamara }\end{array}$ & 109 mm & $115-118 \mathrm{~mm}$ & $117 \mathrm{~mm}$ & $115-118 \mathrm{~mm}$ \\
\hline & $\begin{array}{c}\text { BCA-LM. } \\
\text { Bjork-Jarabak }\end{array}$ & $69 \mathrm{~mm}: 72,5 \mathrm{~mm}$ & 1:01 & 73,3: 77 & 1:01 \\
\hline
\end{tabular}

o: grados, mm: milímetros, SNA: Silla NasionSubespinal, SNB: Silla NasionSupramental, ANB: Subespinal NasionSupramental, BCA: Base Craneal Anterior, LM: Longitud mandibular, *: Los valores de algunas mediciones registran variaciones en la norma de acuerdo con la edad del paciente.

La tercera opción terapéutica fue realizar un tratamiento de ortodoncia fijo. Esto implicaría extracciones dentales para compensar la discrepancia esqueletal. También implicaría la retrusión de los dientes anteriores superiores, manteniendo la proinclinación de los incisivos inferiores. Esta alternativa no mejoraría las condiciones periodontales del arco mandibular, pero, mejoraría parcialmente el perfil facial del paciente.

Debido a la falta de consentimiento y asentimiento de los padres y el paciente respectivamente, para realizar la opción ortodóncico-quirúrgica, se decidió usar un AF removible. Se incluyó una función ortopédica con avance mandibular en el AF-debido a la ausencia de contraindicaciones- mientras se realizaban los movimientos dentales.

El uso de AF como recurso terapéutico proporciona versatilidad, ya que permite realizar acciones ortopédicas y movimientos dentales de forma conjunta ${ }^{(4)}$. Basándose en esta versatilidad, es posible realizar modificaciones e introducir diversos accesorios a los diseños clásicos de AF, para adaptarlos a las necesidades del caso.

Los cambios mandibulares morfogénicos asociados al uso de AF para generar avance mandibular han sido evaluados con diferentes métodos ${ }^{(10,11)}$. Sin embargo, existen controversias respecto del mecanismo biológico por el cual la mandíbula se adapta a una nueva posición, y también en términos de cambios morfológicos y posicionamiento mandibular ${ }^{(6)}$. Además, los efectos de los AF so- 
J. Aylwin Ramírez, A. Hidalgo Rivas, E. Palma Díaz Tratamiento exitoso con activador funcional removible en paciente clase II esqueletal con mordida en tijera, después del peak de crecimiento puberal: caso y revisión de la literatura

bre la ATM siguen siendo objeto de discusión ${ }^{(10,12)}$, debido a los posibles daños que podrían causar a nivel de las articulaciones.

Se sabe que el momento óptimo para iniciar la terapia ortopédica es durante, o ligeramente después del peak de crecimiento puberal ${ }^{(13)}$. Se puede decir entonces, que el uso de AF debe ser indicado en CS3 del $\mathrm{VMI}^{(8)}$. En cualquier caso, se ha descrito que los AF pueden ser exitosos en la etapa CS4, pero con diferentes niveles de efectividad, principalmente dependiendo de la cooperación del paciente en su uso ${ }^{(8)}$. A pesar de que estudios en animales han demostrado que pueden lograrse cambios esqueletales mandibulares aplicando un avance de la mandíbula ${ }^{(14)}$, los efectos en humanos siguen siendo controversiales.

En humanos ha sido difícil establecer la influencia de los AF en el crecimiento mandibular, debido diferencias en los estudios, tales como parámetros evaluados, tamaño muestral, duración del tratamiento, características de grupos control y seguimiento ${ }^{(15)}$. Por otro lado, en humanos existe un problema ético, ya que no es posible detener un tratamiento que pueda ser efectivo en el grupo control.

En relación con los cambios en el crecimiento mandibular, se ha descrito una ligera influencia de los AF de Andressen, Fränkel y Herbst en el crecimiento mandibular ${ }^{(16)}$. Se observó una diferencia en la longitud mandibular de 1,53 $\mathrm{mm}$ en pacientes tratados con AF removibles en comparación con el grupo no tratado ${ }^{(16)}$.Se ha reportado que el AF Sander Bite Jumping produce un mayor aumento de la longitud mandibular $(3,40 \mathrm{~mm})$, seguido en orden decreciente por Twin Block, Bionator, Harvold Activator y Fränkel ${ }^{(16)}$. Sin embargo, otros autores no han mostrado cambios en el crecimiento mandibular ${ }^{(17,18)}$. De la misma manera, no hay consenso sobre los efectos de los AF en el crecimiento mandibular ${ }^{(16,18)}$ También hay diferentes maneras de medir los cambios en el crecimiento mandibular ${ }^{(16)}$. Se han reportado diferencias estadísticamente significativas en el crecimiento mandibular estimulado por $\mathrm{AF}^{(16)}$, cuando se usa el Punto Articular como referencia. Sin embargo, el Punto Articular no es estable, ya que depende de la posición de la mandíbula en el avance mandibular hacia adelante. El Punto Condileon es ideal para medir la eficiencia de los AF en el crecimiento mandibular, y cuando se toma como referencia, no se han reportado diferencias para cualquier tipo de dispositivo ${ }^{(16)}$.

Existen reportes que comparan resultados usando AF fijos y AF removibles. Así, Taki y Ghaffarpasand $^{(17)}$ no encontraron cambios significativos en cuanto a crecimiento o posición maxilar para ambos grupos (AF fijos y removibles). También observaron que la posición mandibular avanzó significativa y favorablemente en ambos grupos. (17). Observaron también aumento de vías respiratorias inferiores significativos y favorables, con el uso de AF removibles y AF fijos ${ }^{(17)}$.

Los AF realizan acciones que ayudan a corregir la clase II esqueletal, como los cambios a nivel condilar $^{(18)}$. Se han reportado cambios en los niveles del factor de transcripción SOX9 en modelos de ratones $^{(19)}$. El SOX9 es una proteína que regula el proceso de diferenciación de los condrocitos en las células mesenquimales a partir de la capa proliferativa de los cóndilos mandibulares en desarrollo ${ }^{(19)}$. El SOX9 también induce la producción de colágeno tipo II por parte de los condrocitos, es decir, la formación de la matriz cartilaginosa. Los niveles más altos de SOX9 se han observado en ratones con avance mandibular - mecanismo usado por los AF - en comparación con ratones sin avance mandibular. Esta diferencia en los niveles de SOX9 indica la causa de una diferenciación temprana de células mesenquimales en condrocitos y una entrada temprana en la ruta condrogénica. Se ha observado que niveles más altos de colágeno tipo II -el más importante dentro de la matriz del cartílago- se alcanzan antes en ratones con avance mandibular ${ }^{(19)}$. Por lo tanto, los AF aceleran el crecimiento condilar, anticipando la entrada y progresión de las células en el proceso de condrogénesis.

El uso de AF mejora la condrogénesis al influir favorablemente en la síntesis de colágeno tipo $\mathrm{II}^{(19)}$. De esta manera, el avance mandibular conduce a la aceleración y a la mejora del crecimiento condilar. La altura del cóndilo aumenta de forma estadísticamente significativa con el uso de AF, y también disminuye el ángulo de la eminencia $\operatorname{articular}^{(19)}$. Con el uso de AF, el disco articular no cambia significativamente su posición, jugando un papel en la remodelación adaptativa, como resultado de estos tratamientos ${ }^{(10)}$.

A nivel del hueso temporal, el avance mandibular con los AF conduce a una mayor formación 
ósea en la fosa mandibular. La mayor cantidad de aposición ósea nueva ocurre en la región posterior de la fosa mandibular ${ }^{(12)}$. La respuesta al avance mandibular muestra que las células mesenquimales en la región posterior de la fosa mandibular están en la dirección de tracción ${ }^{(18)}$. Esta disposición de las células mesenquimales en la dirección de tracción explica la mejora de la aposición ósea en esta región durante el avance mandibular ${ }^{(12)}$. El aparato Twin-Block, por ejemplo, aumenta el volumen condilar, la longitud mandibular y la distancia intercondilar, estimulando el crecimiento condilar hacia arriba y hacia atrás ${ }^{(20)}$. El grado de avance mandibular es un factor relevante para obtener el crecimiento condilar ${ }^{(20)}$. El crecimiento condilar ocurre cuando diferentes niveles de respuestas celulares son desencadenadas por deformaciones mecánicas ${ }^{(20)}$. Los diferentes niveles de respuestas celulares pueden ser producidos a nivel de mediadores de mecanotransducción $\mathrm{IHH}$ (del inglés: Indian Hedgehog Homolog). Así también de reguladores de la madurez celular PTHrP (péptido relacionado con la hormona paratiroidea), y de la cantidad de cartílago (colágeno tipo II). Los diferentes niveles de respuestas celulares se evidencian por el aumento en los niveles de expresión de IHH, PTHrP y colágeno tipo II, resultando en una mayor cantidad de hueso formado ${ }^{(21)}$.

Se ha señalado que debe existir un umbral mínimo de deformación mecánica, que debe superarse para obtener un mayor crecimiento condilar debido a formación de hueso nuevo ${ }^{(21)}$. La formación de hueso, a su vez, se observa en relación con la recuperación morfológica mandibular por regeneración condilar observada en condilectomías de ratones ${ }^{(21)}$. Los cambios histomorfológicos involucrados en la regeneración condilar y su dependencia del tiempo son inciertos ${ }^{(22)}$. Se ha observado en humanos evaluados un año después de las condilectomías, una remodelación ósea condilar con características de cóndilos normales ${ }^{(22)}$. Esta remodelación se produce con formación de hueso cortical, con distancia máxima de $4,5 \mathrm{~mm}$ de la fosa mandibular, similar al efecto de avance mandibular mediado por los AF. Esta respuesta condilar evidencia que la reparación y remodelación condilar un año después de la condilectomía proporcionan una morfología condilar similar a la anatomía normal ${ }^{(23)}$. En el presente caso, los cambios dentarios obtenidos en el tratamiento con AF fueron: corrección de mordida en tijera y arcos dentales conformados y coordinados. También se incorporó el avance mandibular, ya que no había contraindicaciones. Además, se obtuvieron resultados favorables en términos de crecimiento mandibular, con salud y estabilidad de las ATM. Así, en base al caso presentado, se puede sugerir que, en pacientes clase II esqueletal, braquifaciales, aunque no se encuentren en el período de peak puberal, los AF podrían ser utilizados conjuntamente para correcciones dentales y ortopédicas.

Aunque muchos casos tratados con AF requieran una segunda fase de tratamiento con aparatos fijos de ortodoncia, es posible que los objetivos terapéuticos de la fase con aparatos fijos, sean de menor magnitud debido a la acción de los AF.

\section{CONCLUSIÓN}

En el presente caso, el uso de la AF mostró ser útil en el tratamiento de pacientes con mordida en tijera, braquifaciales, clase II esqueletal, después del peak de crecimiento puberal.

Independientemente de las explicaciones sobre el mecanismo que influye en el crecimiento condilar y mandibular, el presente caso permite inferir que se pueden obtener buenos resultados, cuando se usa adecuadamente un AF removible. Los cambios en el crecimiento mandibular pueden ser logrados adicionalmente y simultáneamente a las acciones dentales de los AF.

\section{REFERENCIAS BIBLIOGRÁFICAS}

1. Kannan A, Sathyanarayana HP, Padmanabhan S. Effect of functional appliances on the airway dimensions in patients with skeletal class II malocclusion: A systematic review. J Orthodont Sci 2017; 6:54-64.

2. Dimberg L, Lennartsson B, Arnrup K, Bondemark L. Prevalence and change of malocclusions from primary to early permanent dentition: a longitudinal study. Angle Orthod 2015; 85:728-34.

3. Santamaría-Villegas A, Manrique-Hernandez R, Alvarez-Varela E, Restrepo-Serna C. Effect of removable functional appliances on mandibular length in patients with class II with retrognathism: systematic review and meta-analysis. BMC Oral Health 2017; 17:52. doi: 10.1186/s12903-017-0339-8

4. Koretsi V, Zymperdikas VF, Papageorgiou SN, Papadopoulos MA. Treatment effects of fixed functional appliances in patients with Class II malocclusion: a systematic review and meta-analysis. Eur J Orthod 2015; 37:418-34.

5. Saikoski LZ, Cançado RH, Valarelli FP, de Freitas KM. Den- 
J. Aylwin Ramírez, A. Hidalgo Rivas, E. Palma Díaz Tratamiento exitoso con activador funcional removible en paciente clase II esqueletal con mordida en tijera, después del peak de crecimiento puberal: caso y revisión de la literatura

toskeletal effects of Class II malocclusion treatment with the Twin Block appliance in a Brazilian sample: a prospective study. Dental Press J Orthod 2014; 19:36-45.

6. DiBiase AT, Cobourne MT, Lee RT. The use of functional appliances in contemporary orthodontic practice. $\mathrm{Br}$ Dent J 2015; 218:123-8.

7. Martina R, Cioffi I, Galeotti A, Tagliaferri R, Cimino R, Michelotti A, et al. Efficacy of the Sander bite-jumping appliance in growing patients with mandibular retrusion: a randomized controlled trial. Orthod Craniofac Res 2013; 16:116-26.

8. Angelieri F, Franchi L, Cevidanes LHS, Scanavini M, McNamara J. Long-term treatment effects of the FR-2 appliance: a prospective evaluation 7 years post-treatment. Eur J Orthod 2013; 36:192-9.

9. Franchi L, Baccetti T, McNamara JA Jr. Mandibular growth as related to cervical maturation and body height. Am J Orthod Dentofacial Orthop 2000; 118:335-40.

10. Ma X, Fang B, Dai Q, Xia Y, Mao L, Jiang L. Temporomandibular joint changes after activator appliance therapy: a prospective magnetic resonance imaging study. J Craniofac Surg 2013; $24: 1184-9$

11. Franchi L, Pavoni C, Faltin K, Bigliazzi R, Gazzani F, Cozza P. Thin-plate spline analysis of mandibular shape changes induced by functional appliances in Class II malocclusion: A longterm evaluation. J Orofac Orthop 2016; 77:325-33.

12. Rabie AB, Zhao Z, Shen G, Hägg EU, Robinson W. Osteogenesis in the glenoid fossa in response to mandibular advancement. Am J Orthod Dentofac Orthop 2001; 119:390-400.

13. Wong RW, Alkhal HA, Rabie AB. Use of cervical vertebral maturation to determine skeletal age. Am J Orthod Dentofacial Orthop 2009; 136:484.e1-6.

14. Oksayan R, Sökücü O, Üçüncü N. The effects of low-level laser therapy on condylar growth with a mandibular advancement appliance in rats. Photomed Laser Surg 2015; 33:252-7.

15. Cozza P, Baccetti T, Franchi L, De Toffol L, McNamara J A. Mandibular changes produced by functional appliances in Class II malocclusion: a systematic review. Am J Orthod Den- tofacial Orthop 2006; 129:599.e 1-12.

16. Chen JY, Will LA, Niederman R. Analysis of efficacy of functional appliances on mandibular growth. Am J Orthod Dentofacial Orthop 2002; 122:470-6.

17. Taki AA, Ghaffarpasand A. Effects of functional appliance therapy on the depth of the pharyngeal airways: activator vs. forsus. J Dent Health Oral Disord Ther 2015; 3. doi: 10.15406/ jdhodt.2015.03.00082

18. Rabie AB, She TT, Hägg U. Functional appliance therapy accelerates and enhances condylar growth. Am J Orthod Dentofacial Orthop 2003; 123:40-8.

19. Molina W. Histological changes of the mandibular condyle in the human fetus at early stages of gestation. Int $\mathrm{J}$ Embryol 2014. doi: http://dx.doi.org/10.1155/2014/735949

20. Yildirim E, Karacay S, Erkan M. Condylar response to functional therapy with Twin-Block as shown by cone-beam computed tomography. Angle Orthod 2014; 84:1018-25.

21. Rabie ABM, Al-Kalaly A. Does the degree of advancement during functional appliance therapy matter? Eur $\mathrm{J}$ Orthod 2008; 30:274-82.

22. Nakano M, Fujita T, Ohtani J, Kawata T, Kaku M, Motokawa $M$, et al. Effects of mandibular advancement on growth after condylec-tomy J Dent Res 2009; 88:261-5.

23. Olate S, Cantín M, Palmieri C, Alister JP, Muñoz M, de Moraes M. Mandibular condyle repair after partial condylectomy in patients with active condylar hyperplasia. Int J Morphol 2015; 33:759-63.

\section{RESPONSABLE DEL TRABAJO:}

\section{Edgard Palma Díaz}

Escuela de Odontología

Universidad de Talca

Avenida Lircay S/N

Talca, Chile

Teléfono +56-71-2200476

Correo electrónico: epalma@utalca.cl 